\title{
DIE CHARAKTERISIERUNG UND DIE IDENTIFIZIERUNG VON POLYURETHAN-ELASTOMERFASERN (ELASTHANFASERN) MIT HILFE DER INFRAROTSPEKTROMETRIE
}

Die Polyurethan-Elastomerfasern (neuerlich auch Elasthanfasern genannt) sind, nach DIN 60001, Ausgabe 1970, synthetische Fasern aus solchen (linearen) Hochpolymeren, die zu mindestens 85 Gew.- $\%$ aus segmentiertem Polyurethan bestehen.

Polyurethane werden durch Polyaddition von Diisocyanaten mit Glykolen erhalten. Ein segmentiertes Polymer ist ein Block-Copolymer, in welchem Einheiten unterschiedlicher Zusammensetzung alternieren. Die verschiedenen Blöcke sind in den Polyurethan-Elastomierfasern (Elasthanfasern) «harte» und «weiche» Segmente.

Die Polyurethan-Elastomerfasern bzw. Elasthanfasern gehören zu den Elastofasern (hochelastischen Fasern). Hierunter werden solche Fasern zusammengefaßt, die aufgrund ihres chemischen Aufbaus extrem hoch verformbar sind, d. h. eine Reißdehnung von mehr als $200 \%$, üblicherweise $500-800 \%$, aufweisen und nach Aufheben der Verformungskräfte im wesentlichen augenblicklich und fast vollständig in den ursprünglichen Zustand zurückkehren, d.h. eine sehr hohe elastische Dehnung besitzen [ $\left.{ }^{1}\right]$. Mit dieser Definition werden die elastischen texturierten Garne ausgeschlossen. Andererseits bezieht diese allgemeinere Definition in den Begriff der hochelastischen Fasern auch die Gummifäden aus Kautschuk und andere synthetische Elastofasern ein, die nicht auf Basis von Polyurethan hergestellt sind. Die beiden letzteren werden als Elastodienfasern bezèchnet.

Die Elastodienfasern sind, nach DIN 60001, Ausgabe 1970, Fasern, die aus natürlichem Polyisopren (Gummi) oder synthetischem Polyisopren oder aus solchen Hochpolymeren bestehen, die durch Polymerisation eines Diens oder mehrerer Diene evtl, unter Zusatz eines Vinylmonomers oder mehrerer Vinylmonomere, entstanden sind.

Die Grundlage für die Synthese der heute auf dem Markt befindlichen Polyurethan-Elastomerfasern (Elasthanfasern) ist das im Jahre 1937 aufgefundene Diisocyanat-Polyadditionsverfahren (Polyurethan-Verfahren) $\left[{ }^{1-3}\right]$.

Uber die Ausgangstoffe und die Bildung der Polymeren gibt es eine reichliche Menge von Literaturquellen [ $\left.{ }^{4-8,12}\right]$.

A. Bildung einfacher linearer Polyurethane nach dem DisocyanatPolyadditionsverfahren $\left[{ }^{3}\right]$ :

$$
n \cdot \underset{\text { Glykol }}{-\mathrm{RO}}-\mathrm{\textrm {R } _ { 1 }}-\mathrm{OH}+n \cdot \mathrm{O}=\underset{\text { Dicyan }}{\mathrm{C}}=\underset{\mathrm{N}}{\mathrm{N}}-\mathrm{R}_{2}-\mathrm{N}=\mathrm{O} \rightarrow
$$




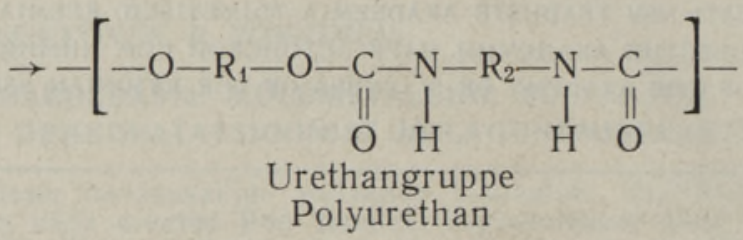

Technisch am leichtesten zugänglich und zugleich für die Faserherstellung am besten brauchbar ist das Polyurethan aus 1,4-Butandiol $\mathrm{HO}\left(\mathrm{CH}_{2}\right)_{4} \mathrm{OH}$ und Hexamethylendiisocyanat (1,6-Hexandiisocyanat) OCN $\left(\mathrm{CH}_{2}\right)_{6} \mathrm{NCO}$, aus welchem die Polyurethanfaser Dorlon als grobes Monofil ersponnen wird.

B. Bildung elastomerer Polyurethane als Block-Polymere [ $\left.{ }^{9}\right]$ (segmentierte Polyurethane).

Hierzu wird bei der Polyadditionsreaktion nach A ein Großteil des Glykols durch langkettige niedrigschmelzende Dihydroxyl-Verbindungen mit Molekularmassen um 2000 ersetzt. Die elastomeren Polyurethane werden in mehreren Stufen aufgebaut. In ihnen alternieren Einheiten unterschiedlicher Konstitution (Block-Copolymere). Die Blöcke werden ihrem Gefüge entsprechend als «weiche» bzw. «harte» Segmente bezeichnet $\left[9,{ }^{0}\right]$; sie sind durch Urethanbindungen verknüpft. Aus Urethanen sind außerdem die «harten» Segmente aufgebaut. Das kristalline «harte» Gefüge des Polyurethans wird durch die amorphen «weichen» Segmente dèr höhermolekularen Dihydroxyl-Verbindung, deren Makromolekülketten innere Beweglichkeit aufweisen, so weit aufgelockert, daß sich solche segmentierte Polyurethane um mehrere Hundert Prozent dehnen lassen [11]. Der Aufbau des «weichen» Segmentes [6,7,11] und die Vorbereitung zum Verknüpfen der Segmente geschehen folgenderweise.

Zuerst wird ein Polymer mit endständigen Hydroxylgruppen, einem hohen Prozentsatz bifunktioneller Anteile (zur späteren Bildung des segmentierten Polymers), niedrigem Schmelzpunkt (unter $50^{\circ} \mathrm{C}$ ) und niedriger Molmasse (zwischen 1000 und 3000), - das Makroglykol gewonnen. Das Makroglykol kann ein Polyätherglykol sein, z. B. Polytetrahydrofuran $\mathrm{H}\left(-\mathrm{O}-\mathrm{CH}_{2}-\mathrm{CH}_{2}-\mathrm{CH}_{2}-\mathrm{CH}_{2}\right) n \mathrm{OH}$ (Teracol) [ $\left.{ }^{3}\right]$, hergestellt durch Polymerisation von Tetrahydrofuran [ $\left.{ }^{12}\right]$, oder ein Polyesterglykol, gewonnen durch Polykondensation einer Dicarbonsäure mit einem geringen Glykolüberschuß, z. B. Adipinsäure + Athylenglykol $\rightarrow$ $\rightarrow$ Polyäthylenadipinatglykol. Auch Mischungen von Glykolen werden verwendet.

Zum Zusammenschließen der Hydroxyl-Endgruppen des Makromoleküls durch Diisocyanate: das Bilden des Präpolymers mit Urethanbindungen zwischen den «weichen» und den zukünftigen «harten» Segmenten und mit Isocyanatgruppen - des Makrodiisocyanats.

Für diese Reaktion werden bevorzugt aromatische Diisocyanate herangezogen, insbesondere Diphenylmethan- $4,4^{\prime}$-Diisocyanat $\left[{ }^{13}\right]$

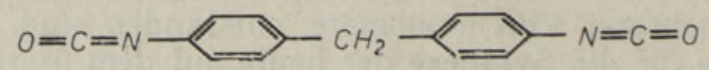

oder isomere Toluylendiisocyanate

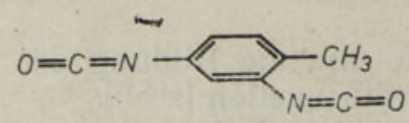

Die Diisocyanate werden jeweils im Uberschuß zum Makromolekül eingesetzt (3:2 oder $2: 1$ ); Verlängern der Ketten und Bilden des «harten» Segmentes (Polyadditions-Reaktion) [ $\left.{ }^{6}\right]$.

Das Präpolymer (Makrodiisocyanat) wird in einem hochpolaren orga- 
nischen Lösungsmittel, z.B. Dimethylformamid oder Dimethylacetamid aufgelöst und mit einem niedermolekularen Diamin oder Diol (letztere im Uberschuß) als Kettenverlängerer zur Reaktion gebracht. (Diaminverlängerung durch Athylendiamin, m-Xylylendiamin oder Hydrazin; Glykolverlängerung durch Athylenglykol.)

Die «harten» Segmente [ $\left.{ }^{11}\right]$ bestehen aus den verlängerten Diisocyanaten.

Herstellungsverfahren der Fasern [ $\left.{ }^{7,11}, 12\right]$. Das Erspinnen von Polyurethan-Elastomerfasern (Elasthanfasern) kann mittels verschiedener Verfahren erfolgen.

a) Trockenspinnen des in der Lösung vorliegenden elastomeren segmentierten Polyurethans durch Auspressen durch eine Mehrlochdüse in ein senkrecht stehendes, beheiztes Rohr, in welchem das Lösungsmittel den Fäden durch Ausdampfen entzogen wird.

b) $\mathrm{NaBspinnen} \mathrm{der} \mathrm{Polyurethan-Elastomer-Lösung} \mathrm{aus} \mathrm{der} \mathrm{Düse} \mathrm{in} \mathrm{ein}$ Wasserbad oder in eine wässrige Dimethylformamidlösung, in welcher der Faden durch Entzug des Lösungsmittels koaguliert.

c) Chemisches Spinnen («Reaktiv-Spinnen»), indem die Makrodiisocyanat-Schmelze oder -Lösung, d. h. das Präpolymer vor der Kettenverlängerung ausgesponnen und erst im Spinnbad nach der Bildung des Fadens, mit dem Diamin (als Kettenverlängerer) in wässriger Lösung zur Reaktion gebracht wird.

d) Thermoplastisches Verformen eines zähflüssigen Polyurethans in einem Extruder durch Düsenöffnungen zu groben Monofilen, die durch Abkühlen erstarren.

Hierüber können Fäden aus durch Schwefel vernetzten Polyurethanen auch durch Schneiden aus langen Elastomerbahnen gewonnen werden. In diesem sind die Querschnitte der Fäden quadratisch oder rechteckig.

Chemische Konstitution. Ein lineares elastomeres Polyurethan-BlockCopolymerisat kann z. B. wie folgt aufgebaut sein $\left[{ }^{14}\right]$ :

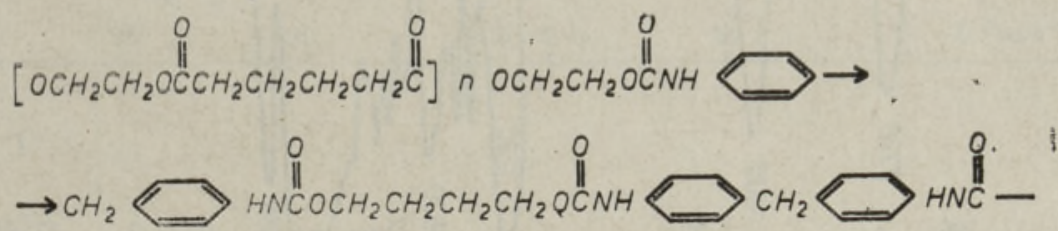

Zur Erkennung und Unterscheidung von Elasthanfasern wurden ausgewählte Lösungsmittel von P.-A. Koch und A. Nettesheim $\left[{ }^{15}\right]$ verwendet. Für eine Erkennung einzelner Fabrikate ergaben sich trotz Prüfung mit verschiedenartigen Säuren und Lösungsmitteln nur wenig charakteristische Merkmale. Ein Reagens, das Polyätherurethanfasern von Polyesterurethanfäsern unterscheiden läßt, ließ sicht nicht finden. Die Feststellung der Zugehörigkeit eines Fabrikats zur Klasse der Polyätherbzw. Polyesterurethane ist nur möglich mittels des Infrarotspektrums $\left[{ }^{16,17}\right]$. Die Gaschromatographie der Pyrolyseprodukte von Elasthanfa. sern haben $O$. Kirret und E. Küllik untersucht $\left[{ }^{18}\right]$.

Das Obenstehende ist aus $\left[{ }^{14}\right]$ entnommen. In unseren früheren Aufsätzen $\left[{ }^{19-23}\right]$ konnten wir zeigen, daB die infrarotspektroskopische Methode zur Charakterisierung und mit Hilfe des Computers für die Identifizierung von Chemie- und Naturfasern anwendbar ist. Das Ziel der vorliegenden Arbeit ist, festzustellen, ob die zuletzt genannte Methode auch die Erkennung der Polyurethan-Elastomerfasern ermöglicht.

Die Infrarotspektren wurden mit dem Specord 75 IR aufgenommen 


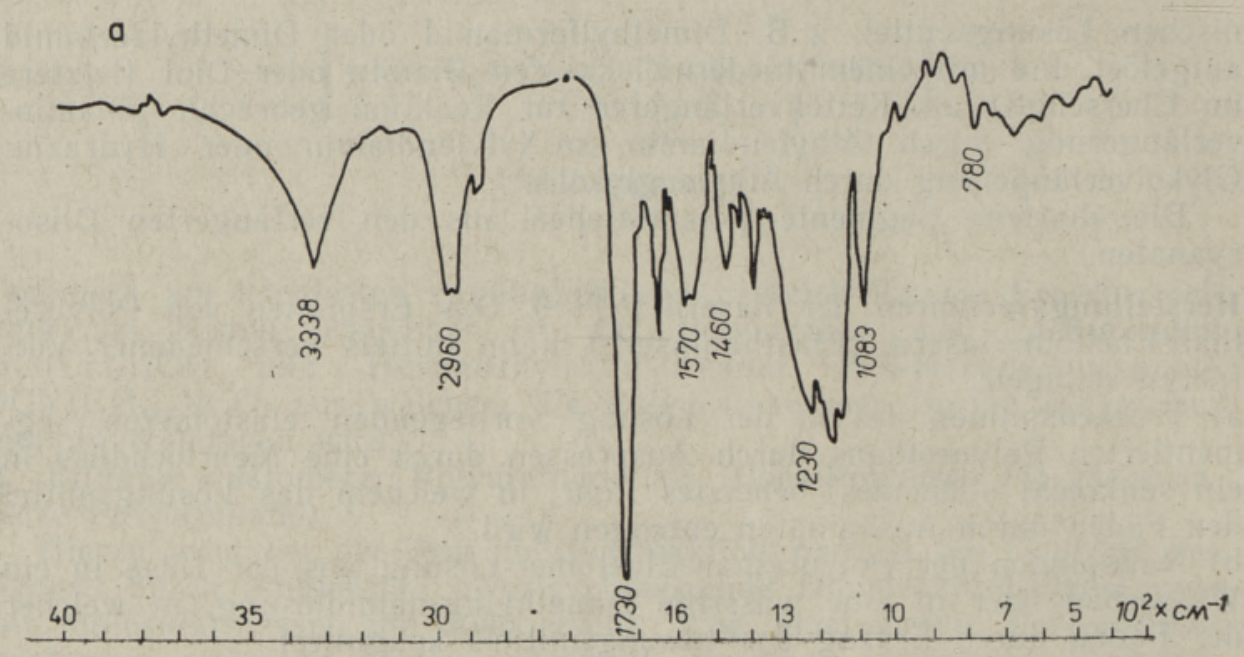

3

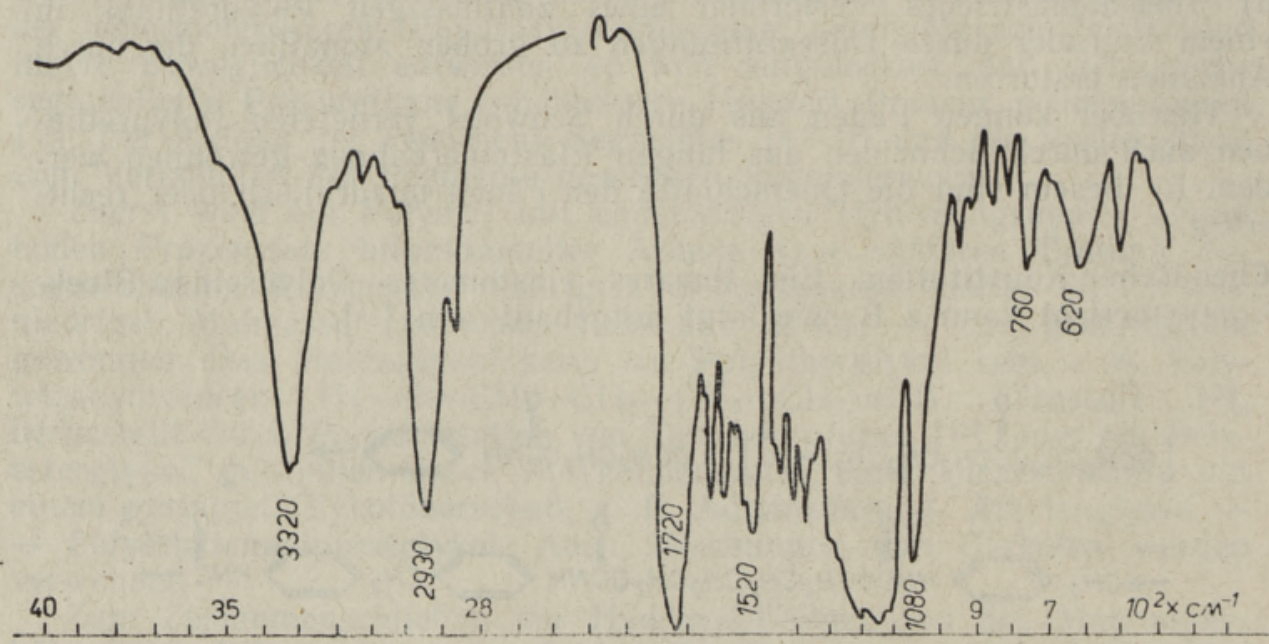

c

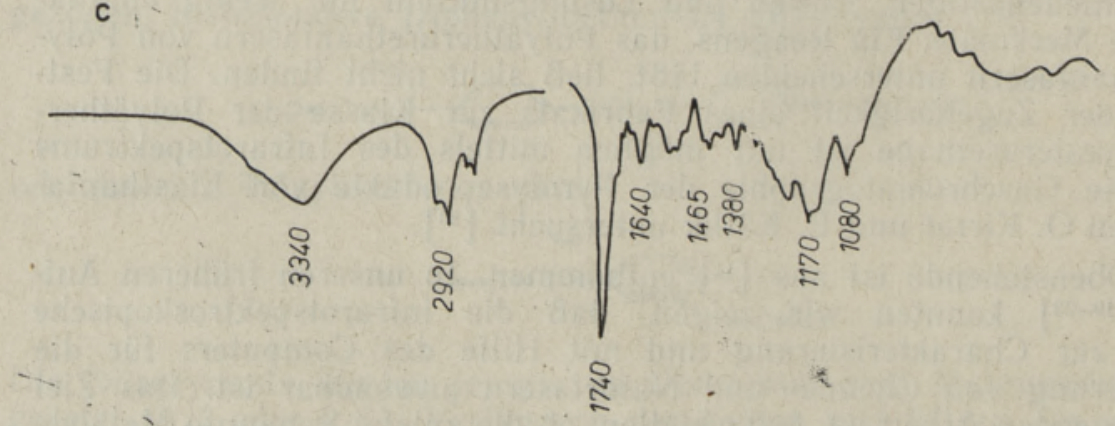

40 

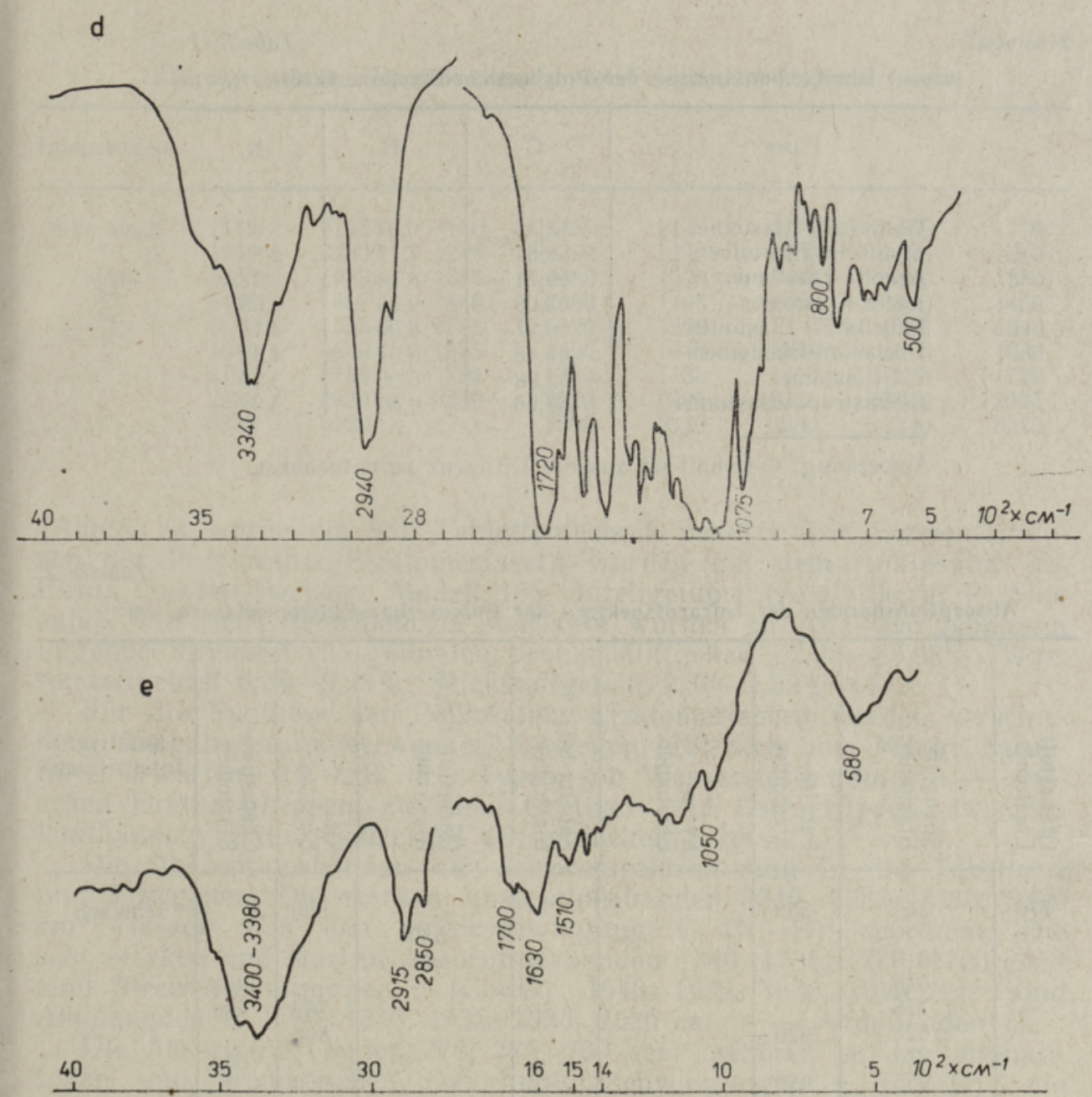

f

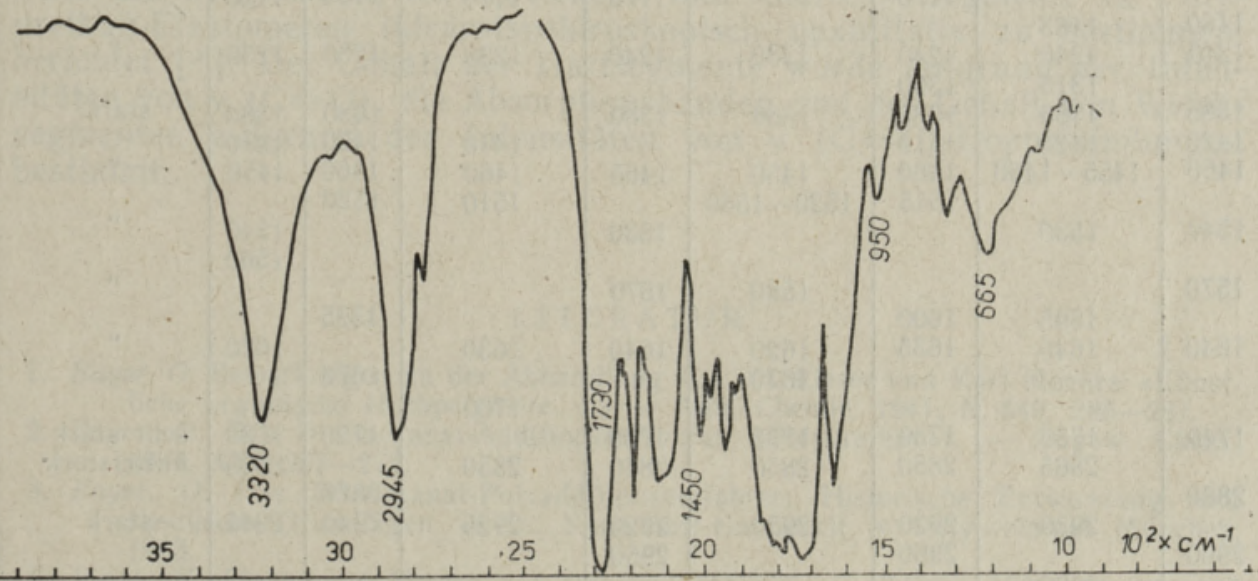

Die Infrarotspektren der Polyurethan-Elastomerfasern: $a-$ Glanzstoff-Elastomer, $b-$ Spanzelle-Elastomer, $c-$ Fabelta-Elastomer, $d-$ Fiz-Elastomer, $e-$ Dorlastan-Elastomer, $f-$ Chemstrand-Elastomer. 
Tabelle 1

Die Elementanalyse der Polyurethan-Elastomerfasern, \%

\begin{tabular}{l|c|c|c}
\hline \multicolumn{1}{c|}{ Faser } & C & H & N \\
& & & \\
\hline & & & \\
Glanzstoff-Elastomer & 53,35 & 6,87 & 3,69 \\
Spanzelle-Elastomer & 56,67 & 7,17 & 2,89 \\
Fabelta-Elastomer & 59,71 & 8,14 & 3,18 \\
Lycra-Elastomer & 63,08 & 9,28 & 3,09 \\
Fabelta-Fil-Elastomer & 60,27 & 8,06 & 3,13 \\
Dorlastan-Elastomer & 60,48 & 8,07 & 4,32 \\
Fiz-Elastomer & 52,98 & 6,26 & 3,26 \\
Chemstrand-Elastomer & 53,96 & 6,80 & 3,67 \\
\end{tabular}

Anmerkung: O-Gehalt ist aus der Differenz zu entnehmen.

Tabelle 2

Absorptionsbanden der Infrarotspektren der Polyurethan-Elastomerfasern, $\mathbf{c m}^{-1}$

\begin{tabular}{|c|c|c|c|c|c|c|c|c|}
\hline 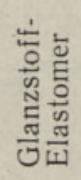 & 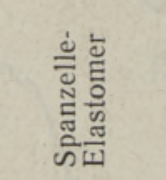 & 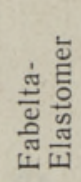 & 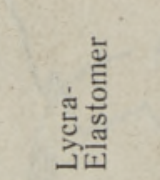 & 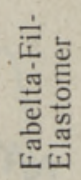 & 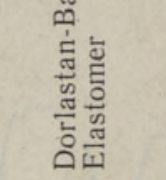 & 这㝕 & 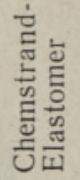 & $\begin{array}{c}\text { Relative } \\
\text { Intensitäten }\end{array}$ \\
\hline 770 & $\begin{array}{l}545 \\
\\
615 \\
645 \\
775 \\
825 \\
870 \\
955\end{array}$ & $\begin{array}{l}820 \\
950\end{array}$ & & & 580 & $\begin{array}{l}760 \\
800 \\
900\end{array}$ & $\begin{array}{l}665 \\
760 \\
880\end{array}$ & $\begin{array}{c}\text { schwach } \\
\text { " } \\
" \\
" \\
"\end{array}$ \\
\hline 1083 & 1085 & 1080 & $\begin{array}{l}1040 \\
1080 \\
1140\end{array}$ & $\begin{array}{l}1080 \\
1130\end{array}$ & 1050 & $\begin{array}{r}940 \\
1025 \\
1130\end{array}$ & $\begin{array}{r}990 \\
1070 \\
1: 40\end{array}$ & $\begin{array}{c}" \\
\text { stark } \\
\text { sehr stark }\end{array}$ \\
\hline 1145 & 1147 & $\begin{array}{l}1150 \\
1170\end{array}$ & 1160 & 1170 & 1170 & & 1170 & ", \\
\hline $\begin{array}{l}1180 \\
1240\end{array}$ & $\begin{array}{l}1183 \\
1240 \\
1315\end{array}$ & $\begin{array}{l}1240 \\
1300\end{array}$ & 1230 & 1240 & 1230 & 1230 & 1230 & $"$ \\
\hline $\begin{array}{l}1383 \\
1420\end{array}$ & $\begin{array}{l}1383 \\
1420\end{array}$ & $\begin{array}{l}1380 \\
1410\end{array}$ & 1380 & 1380 & 1410 & $\begin{array}{l}1380 \\
1400\end{array}$ & $\begin{array}{l}1380 \\
1410\end{array}$ & stark \\
\hline 1460 & $1455-1460$ & $\begin{array}{l}1460 \\
1515\end{array}$ & $\begin{array}{c}1450 \\
1520-1580\end{array}$ & 1465 & $\begin{array}{l}1460 \\
1510\end{array}$ & $\begin{array}{l}1460 \\
1520\end{array}$ & 1450 & $\begin{array}{l}" \\
,\end{array}$ \\
\hline $\begin{array}{l}1540 \\
1570\end{array}$ & 1530 & & 1580 & $\begin{array}{l}1530 \\
1570\end{array}$ & & & $\begin{array}{l}1540 \\
1560\end{array}$ & $"$ \\
\hline 1640 & $\begin{array}{l}1595 \\
1640\end{array}$ & $\begin{array}{l}1600 \\
1635\end{array}$ & $\begin{array}{l}1620 \\
1670\end{array}$ & $\cdot 1640$ & 1630 & $\begin{array}{l}1595 \\
1670\end{array}$ & 1620 & $"$ \\
\hline 1740 & $\begin{array}{l}1735 \\
2865\end{array}$ & $\begin{array}{l}1730 \\
2850\end{array}$ & $\begin{array}{l}1730 \\
2850\end{array}$ & $\begin{array}{l}1730 \\
2860\end{array}$ & 2850 & 1720 & $\begin{array}{l}1730 \\
2865\end{array}$ & $\begin{array}{l}\text { sehr stark } \\
\text { mittelstark }\end{array}$ \\
\hline 2960 & 2930 & $\begin{array}{l}2920 \\
2960\end{array}$ & 2920 & $\begin{array}{l}2920 \\
2960\end{array}$ & 2925 & 2940 & 2945 & stark \\
\hline 3338 & $\begin{array}{l}3020 \\
3110 \\
3320\end{array}$ & 3420 & $\begin{array}{l}3330 \\
3430\end{array}$ & 3340 & $3380-3400$ & $\begin{array}{l}3110 \\
3340 \\
\\
3425\end{array}$ & $\begin{array}{l}3100 \\
3320\end{array}$ & stark \\
\hline
\end{tabular}


Charakteristische Absorptionsbanden der Polyurethan-Elastomerfasern

Intensitäten

\begin{tabular}{ccccccccc}
\hline & & & & & & 1170 & 1165 & 1170 \\
sehr stark & 1145 & 1147 & 1150 & 1150 & 1170 & 1170 & 1230 & 1230 \\
stark & 1240 & 1240 & 1240 & 1230 & 1240 & 1230 & 1380 & 1380 \\
$"$ & 1383 & 1383 & 1380 & 1380 & 1380 & 1380 & 1380 & 1450 \\
$\#$ & 1460 & 1457 & 1460 & 1450 & 1465 & 1460 & 1460 & 1540 \\
$\#$ & 1540 & 1530 & 1515 & 1520 & 1530 & & 1520 & 1630 \\
$"$ & 1640 & 1640 & 1635 & 1620 & 1640 & 1630 & 1670 & 1730 \\
$"$ & 1740 & 1735 & 1730 & 1730 & 1730 & 1720 & 1720 & 2945 \\
$"$ & 2960 & 2930 & 2960 & 2920 & 2920 & 2925 & 2940 & 3320
\end{tabular}

(Abb.). Es wurde die $\mathrm{KBr}-\mathrm{Tablettentechnik} \mathrm{benutzt.} \mathrm{Die} \mathrm{Elementanaly-}$ sen der Polyurethan-Elastomerfasern wurden mit dem Analysator der Firma Hewlett-Packard, Modell 185, durchgeführt (Analytikerin R. Soosalu). Für acht verschiedene Fabrikate wurden in Analyseergebnissen folgende Streubereiche gefunden: Kohlenstoffgehalt 52,98-63,08\%; Wasserstoffgehalt $6,26-9,28 \%$; Stickstoffgehalt $2,89-4,32 \%$ (Tab. 1).

Für die Synthese der Polyurethan-Elastomerfasern werden verschiedene Ausgangsstoffe verwendet, deswegen läßt sich eine Menge Strukturen erwarten, wie z. B. drei Typen von Wasserstoffbindungen - zwischen Urethangruppen, zwischen Urethan- und Estergruppen, zwischen Urethangruppe und Sauerstoff des einfachen Esters.

Die Absorptionsbanden der Infrarotspektren sind in der Tabelle 2 und 3 gegeben. Die starken Absorptionsbanden 3340, 3338, 3330, 3320 $\mathrm{cm}^{-1}$ lassen sich der Streckschwingung $v(\mathrm{~N}-\mathrm{H})$ zuordnen. Die sehr starken und starken Absorptionsbanden 1740, 1735, 1730, $1720 \mathrm{~cm}^{-1}$ sind Streckschwingungen $v(C=O) ; 1640,1635,1630,1620 \mathrm{~cm}^{-1}$ sind Amidbanden I; 1560, 1540, 1535, 1530, $1520 \mathrm{~cm}^{-1}$ - Amidbanden II.

Die Absorptionsbanden $770,775,760 \mathrm{~cm}^{-1}$ gehören $\mathrm{zu}$ den aliphatischen lind aromatischen Deformationsschwingungen $\gamma \quad(C=O)$; die Absorptionsbanden 1240, $1230 \mathrm{~cm}^{-1}$ - zu den Biegeschwingungen $\delta$ $(\mathrm{N}-\mathrm{H})$.

Das Vorhergesagte steht im Einklang mit den Literaturangaben [24, 25]. Uber die Möglichkeit, die «weichen» und «harten» Segmente in Polyurethan-Elastomeren infrarotspektroskopisch quantitativ zu bestimmen, berichtet $\left[{ }^{25}\right]$. Der Gehalt der Hartsegmente wurde aufgrund der Intensitäten von $v(\mathrm{C}=\mathrm{O})$, die Absorptionsbanden und der Gehalt von Weichsegmenten aufgrund der Intensitäten von $v(C=C)$-Doppelbindungen bestimmt.

\section{LITERATUR}

1. Bayer, O. Bemerkungen zu der Abhandlung von Th. Lieser und Karl Macura: «Künstliche organische Hochpolymere. I.» - Ann. Chemie, 1941, N 549, 286-287.

2. Bayer, O. Das Di-Isocyanat-Additionsverfahren (Polyurethane). - Angew. Chem., 1947, A59, 257-272.

3. Bayer, $O$. Das Diisocyanat-Polyadditionsverfahren. Historische Entwicklung und chemische Grundlagen. - In: Kunststoff-Handbuch, 8: Polyurethane. München, 1963.

4. Rinke, H. Elastomere Fasern auf Polyurethanbasis. - Chimia, 1962, 16, 93-105 bzw. Angew. Chem., 1962, 74, 612-617.

5. Bamford, C. H. Polymers and fibers. - J. Text. Inst., 1965, 56, P73-P8s.

6. Ultee, A. J. Spandex-Fasern: Bildung, Struktur und Eigenschaften. - Z. ges. Text.-Ind., 1965, 67, 453-459. 
7. Rinke, H. Polyurethane als Grundlage für elastomere Fäden. - Chimia, 1968, 22, $164-175$.

8. Oertel, $H$. Struktur, Modifiziermöglichkeiten und Eigenschaften segmentierter PU-Elastomer-Filamentgarne. - Chemiefasern/Textilindustrie, 1977, 27/79, $1090-1092,1095,1096 ; 1978,28 / 80,44-49$.

9. Shivers, J. C., Seaman, R. E. Elastic textile fibers based on condensation polymers. I. und II. Vorträge vor der: Gordon Research Conference, Textile Week, July 1958, Colby College, New London, N. H. Als Manuskript gedruckt - El. Du Pont de Nemours Co., Inc., Wilmington, Del.

10. Kolb, H. J. «Lycra». Vortrag vor der: Rubber Section of Washington in Fort Belvoir, March 8, 1961. Als Manuskript gedruckt - El. Du Pont de Nemours Co., Inc., Wilmington, Del.

11. Oertel, $H$. Bayer-Elastomer-Fäden-Aufbau und Eigenschaften. - Bayer-Farben-Revue, 1966, Heft 11.

12. Lyssy, Th. Elastomere Spinnstoffe. - Lenzing. Ber. 1963, Okt., Heft 15, 22-23 bzw. Chemiefasern, 1963, 13, 768-772, 774-776.

13. Oertel, $H$. Elastomere Fäden auf Polyurethanbasis, ihr Aufbau, ihre Eigenschaften, ihre Verwendung. - Melliand Textilber., 1965, 46, 51-59.

14. Koch, P.-A. Elasthanfasern. - Chemiefasern/Textilindustrie, 1979, Febr., 95.

15. Koch, P.-A., Nettesheim, A. Unterscheidungsmöglichkeiten für Polyurethan-Elastomerfasern. Institut für Textiltechnik TH Aachen. Manuskript nicht veröffentlicht.

16. Heidemann, G., Jellinek, G., Ringens, W. Zur Struktur von Polyurethan-Elastomerfasern. - Kolloid-Z. und Z. Polym., 1967, 221, Heft 2, 119-121.

17. Wegener, W., Merkle, R., Berndt, H.-J. Infrarotspektroskopische Identifizierung von elastomeren Faserstoffen mit Hilfe der FMIR-Technik. Institut für Textiltechnik TH Aachen. Manuskript nicht veröffentlicht.

18. Kirret, O., Küllik, E. Analyse der Polyurethan-Elastomerfasern mittels der Pyrolyse-Gaschromatographie. - Z. ges. Text.-Ind., 1969, N 71, 169-171; Изв. АН ЭССР. Хим. Геол., 1969, 18, № 3, 211-217.

19. Kirret, O., Pank, M., Lahe, L. Characterization and identification of polyester fibres and their modifications by infrared spectrometric method. - ENSV TA Toim. Keemia, 1980, 29, N 2, 92-96.

20. Kirret, O., Koch, P.-A., Lahe, L. Characterization and identification of polyamide fibres by infrared spectrometric method. - ENSV TA Toim. Keemia, 1981, 30 , N 4, 280-287.

21. Kirret, $O$., Koch, $P_{\text {. }}$ A., Lahe, $L$. Characterization of polyvinyl chloride fibres by infrared spectroscopic method. - ENSV TA Toim. Keemia, 1982, 31, N 1, $50-53$.

22. Kirret, O., Koch, P.-A., Lahe, L. Characterization and identification of polyacrylonitrile fibres and their modifications and modacrylic fibres by infrared spectrometric method. - ENSV TA Toim. Keemia, 1982, 31, N 3, 197-203.

23. Kirret, O., Lahe, L., Rajalo, G., Kirjanen, E. Characterization and identification of chemical fibres by infrared spectrometric method and computer. - ENSV TA Toim. Keemia, 1983, 32, N 2, 119-124.

24. Hummel/Scholl. Atlas der Kunststoff-Analyse, B. 1, Teil I, München, 1968, 186-187.

25. Yu, M., MacKnight, W. J., Chen, C. H. Y., Thomas, E. L. Structure and morphology of segmental polyurethanes. - Polymer, 1983, 24, Oct., 1328-1329.

\author{
Institut für Chemie \\ der Akademie der Wissenschaften \\ der Estnischen SSR
}

Krefeld-Traar, BRD
Eingegangen

am 26. April 1984

\section{POLUURETAAN-ELASTOMEERKIUDUDE (ELASTAANKIUDUDE) ISELOOMUSTAMINE JA IDENTIFITSEERIMINE INFRAPUNASE SPEKTROMEETRIA ABIL}

Tavaliste polüuretaankiudude kõrval on tähelepanuväärseteks keemilisteks kiududeks kujunenud polüuretaan-elastomeerkiud, uuema nimetusega elastaankiud. Need on valmistatud sünteesil saadud plokkpolümeeridest, mille lineaarses ahelas vahelduvad keemiliselt koostiselt 'erinevad kõvad (jäigad) ja pehmed segmendid.

Polüuretaan-elastomeerkiudude, nagu teistegi keemiliste ja looduslike kiudude isəloomustamiseks saab kasutada infrapunastes spektrites esinevaid väga tugevaid, tugevaid ja keskmise tugevusega absorptsiooniribasid. 
О. КИРРЕТ, П.-А. КОХ, Лилья ЛАХХЕ

\section{ХАРАКТЕРИСТИКА И ИДЕНТИФИКАЦИЯ ПОЛИУРЕТАН-ЭЛАСТОМЕРНЫХ (ЭЛАСТАНОВЫХ) ВОЛОКОН МЕТОДОМ ИК-СПЕКТРОМЕТРИИ}

Исследуются полиуретан-эластомерные волокна, получаемые формованием из синтезированных блокполимеров, в линеарных цепях которых чередуются твердые и мягкие сегменты, отличающиеся друг от друга химическим составом. Дается характеристика этим волокнам на основе очень сильных, сильных и средних полос поглощения в их ИК-спектрах. 